\title{
Design and Analysis of Slotted Sectoral Waveguide Array Antennas Embedded in Cylindrically Stratified Media
}

\author{
Mert Kalfa* and Vakur B. Ertürk \\ Dept. of Electrical and Electronics Engineering, \\ Bilkent University, TR-06800, Bilkent, Ankara, Turkey
}

Slotted waveguide antennas are being widely used in military and commercial applications for many decades. Low cross-polarization, high power capacity, ease of fabrication in microwave bands, and the ability to form arrays make them excellent candidates for phased array antennas in radar applications. However, due to slots being highly resonant (narrow-band, high Q) radiators, their design parameters are very sensitive; hence, accurate design and analysis methods are required for a successful antenna design. Moreover, slotted waveguide array antennas are low-profile structures, which makes them suitable candidates for conformal and structure-integrated applications. Conformal and structure-integrated system solutions are especially required for air platforms, where aerodynamics, radar crosssection (RCS) and efficient use of real estate are of utmost importance. Although the accurate and efficient design and analysis of low-profile conformal slotted waveguide arrays are of great interest, available solution methods in the literature usually suffer in terms of efficiency and memory requirements. Among the available solution methods, one of the widely used solvers are integral equation (IE) based ones that utilize the method of moments (MoM). However, IE solvers suffer from long matrix fill times, especially for matrix entries related to the cylindrically stratified media.

In this study a slotted sectoral waveguide array antenna, covered by multiple dielectric layers (which may represent monolithic or sandwich radomes) is analyzed with a hybrid MoM/Green's function technique in the space domain. Only the fundamental mode $\left(\mathrm{TE}_{11}\right)$ is assumed to be excited inside the waveguide. The longitudinal slots are cut on the broadside wall of the waveguide and are very short in the transverse direction, so that longitudinal electric fields along the slots can be neglected. Using equivalence theorem, the slots are represented with fictitious magnetic current sources, which are expanded by piecewise sinusoid (PWS) basis functions. The magnetic field integral equation (MFIE), resulting from enforcing the continuity of the tangential magnetic fields, is converted into matrix form using Galerkin's procedure. The Green's function representations for the waveguide interior and exterior, which were previously developed by the authors, are utilized to compute the entries of the MFIE matrices and vectors. Then the equivalent slot magnetic currents are computed by solving the matrix equation using iterative matrix inversion techniques. However, the equivalent currents by themselves cannot be used to directly assess the antenna performance. Hence, post-processing parameters such as the $\mathrm{Y} / \mathrm{Z} / \mathrm{S}$-parameters and far-zone radiation patterns are also calculated to have a more detailed evaluation of the antenna.

Analysis results for a generic slotted sectoral waveguide array will be presented, and will be compared to the results obtained from the commercially available full-wave electromagnetic solvers. Additionally, a design methodology for slotted sectoral waveguide arrays is developed using the proposed analysis method, which will also be presented. 